\section{Comparison of Empiric Versus Dosimetry-Guided Radioiodine Therapy: The Devil Is in the Details}

TO THE EDITOR: We read with interest the article by Deandreis et al. (1) that compared a fixed-activity approach to radioiodine treatment of metastatic differentiated thyroid cancer with a method based on whole-body (blood clearance) dosimetry. Similar survival was seen for both cohorts. This study highlights the continuing uncertainty regarding the lack of an optimal approach to treatment for the highestrisk patients, as recognized by both European Association of Nuclear Medicine and American Thyroid Association guidelines $(2,3)$, and demonstrates the difficulty of performing retrospective analyses. It is notable that despite the apparently substantial differences in treatment regimens and patient cohort characteristics between the two centers, a personalized approach was taken in all cases, with patients in both cohorts receiving highly variable levels of cumulated activity, numbers of treatments, and intervals between administrations. Patient follow-up varied from 5 mo to $31 \mathrm{y}$. The paper shows that a highly personalized approach is, in oncologic terms, extremely successful in considerably extending the life-span of patients with distant metastases. Likely because of this great success, the comparatively smaller differences, if they exist, between the different approaches to personalization may have been obfuscated.

This article appears precisely $80 \mathrm{y}$ after the initial development of radioiodine. The ablation of remnant thyroid after thyroidectomy and the treatment of persistent thyroid disease and distant metastases is surely one of the great success stories of cancer management. The pioneering work of clinician Saul Hertz and physicists Karl Compton and Arthur Roberts, after a luncheon talk entitled "What Physics Can Do for Biology and Medicine" by Dr. Compton in November 1936, demonstrated the enormous potential of the fusion of nuclear physics and medicine and led directly to what is possibly the closest conceivable approach to the magic bullet for cancer (4). Initial studies recognized that the effect of radiation on either healthy or malignant tissue depends on the amount of radiation delivered, and more than $10 \mathrm{y}$ before the development of the Anger camera, great efforts were made to calculate the absorbed doses (in Gy) delivered to thyroid metastases and to healthy organs (5). The work led to the formation of the Radioactive Isotope Research Institute in Boston in September 1946, with Dr. Saul Hertz as the director and Dr. Samuel M. Seidlin as the associate director. In the seminal paper by Seidlin et al. (6) concerning treatment of metastatic thyroid cancer, an empiric activity of $3,700 \mathrm{MBq}(100 \mathrm{mCi})$ of ${ }^{130} \mathrm{I}-\mathrm{NaI}$ was administered concomitantly with $760 \mathrm{MBq}$ of ${ }^{131} \mathrm{I}-\mathrm{NaI}$ to deliver 90 Gy to the tumor. ${ }^{130} \mathrm{I}-\mathrm{NaI}$ was found to cause depression of leukocytes, and future administrations settled on treatment solely with ${ }^{131} \mathrm{I}-\mathrm{NaI}$, although still with an administered activity of 3,700 MBq.

This fortuitous combination of the "magic number" with the "magic bullet" paved the way for the use of radiotherapeutics, and the paradigm was applied to most further radiotherapeutics as they were developed. An activity of $3,700 \mathrm{MBq}$, or multiples thereof, was subsequently administered for the treatment of adult and pediatric neuroendocrine tumors using ${ }^{131} \mathrm{I}$-metaiodobenzylguanidine, ${ }^{90} \mathrm{Y}$-DOTATOC, or ${ }^{177} \mathrm{Lu}$ DOTATATE; for the initial treatment of liver metastases using ${ }^{90} \mathrm{Y}$

COPYRIGHT @ 2017 by the Society of Nuclear Medicine and Molecular Imaging. microspheres; and, more recently, for the treatment of bone metastases from prostate cancer using ${ }^{177} \mathrm{Lu}$-prostate-specific membrane antigen.

Highly successful outcomes were reported in the article by Deandreis et al. (1), without apparent correlations either with the whole-body (blood) absorbed doses or with the levels of activity administered. These factors are undoubtedly important but may not be sufficient to generate the improved outcomes that must be available with a more scientific approach. In a dawning era of personalized and precision medicine, radioiodine treatment of differentiated thyroid cancer affords the opportunity to realize the full potential of an individualized approach to treatment that may result in significant patient benefit. This goal can be tackled only by close collaborations between clinicians and medical physicists based on the increasing evidence that outcome depends on the radiation doses delivered rather than on the activities administered (7). The birth of nuclear medicine was blessed with a phenomenally successful cancer treatment by the visionary work of Hertz, Compton, and Roberts. It is surely time to capitalize on their legacy and further improve the treatment-particularly for high-risk and pediatric patients - with the application of imaging and lesion dosimetry in prospective multicenter clinical trials.

\section{REFERENCES}

1. Deandreis D, Rubino C, Tala H, et al. Comparison of empiric versus whole-body/ -blood clearance dosimetry-based approach to radioactive iodine treatment in patients with metastases from differentiated thyroid cancer. J Nucl Med. 2017; 58:717-722.

2. Haugen BR, Alexander EK, Bible KC, et al. American Thyroid Association management guidelines for adult patients with thyroid nodules and differentiated thyroid cancer: the American Thyroid Association guidelines task force on thyroid nodules and differentiated thyroid cancer. Thyroid. 2016;26:1-133.

3. Luster M, Clarke SE, Dietlein M, et al. Guidelines for radioiodine therapy of differentiated thyroid cancer. Eur J Nucl Med Mol Imaging. 2008;35:1941-1959.

4. Hertz B. Dr. Saul Hertz (1905-1950) discovers the medical uses of radioactive iodine: the first targeted cancer therapy. In: Ahmadzadehfar H, ed. Thyroid Cancer: Advances in Diagnosis and Therapy. Rijeka, Croatia: In Tech; 2016.

5. Hertz S, Roberts A. Radioactive iodine in the study of thyroid physiology: the use of radioactive iodine therapy in hyperthyroidism. J Am Med Assoc. 1946;131:81-86.

6. Seidlin SM, Marinelli LD, Oshry E. Radioactive iodine therapy; effect on functioning metastases of adenocarcinoma of the thyroid. J Am Med Assoc. 1946;132:838-847.

7. Strigari L, Konijnenberg M, Chiesa C, et al. The evidence base for the use of internal dosimetry in the clinical practice of molecular radiotherapy. Eur $\mathrm{J} \mathrm{Nucl}$ Med Mol Imaging 2014;41:1976-1988.

\section{Glenn D. Flux* \\ Frederik A. Verburg \\ Carlo Chiesa \\ Manuel Bardiès \\ Katarina Sjögreen Gleisner \\ Barbara Hertz \\ Mark Konijnenberg \\ Michael Lassmann \\ Michael Ljungberg \\ Markus Luster \\ Michael Stabin \\ Lidia Strigari \\ *Royal Marsden Hospital Downs Rd. \\ Sutton, Su SM2 5PT, United Kingdom \\ E-mail: glenn.fux@icr.ac.uk}

Published online Feb. 9, 2017.

DOI: 10.2967/jnumed.116.186643 